\title{
Efiects of Human Genetic Factors (Ethnicity and Race) on Clinical Severity of SARS-COV-2 (COVIID-19)
}

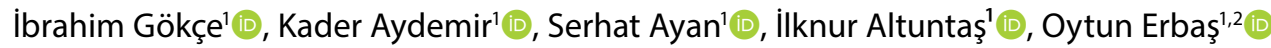

SARS-CoV-2 is the seventh kind of coronavirus that is encountered and leads to a variety of outcomes ranging from the common cold to acute respiratory distress syndrome (ARDS) and sepsis. SARS-CoV-2 first appeared in Wuhan, China at the end of 2019. It has been continuing to spread all around the world by 2020 . Since especially young people show no symptoms (asymptomatic), SARS-CoV-2 has spread quickly all around the world and led to pandemic. Currently, besides there is no definitive treatment for COVID-19, it is thought that genetic diversity and ethnicity may have a role in the clinical course of COVID-19. Analyzing the meta-analysis published in The Lancet Journal, it can be clearly seen that Asian, Hispanic, and Black populations are more likely to be infected with SARS-CoV-2. ${ }^{[1]}$ On the other hand, Analysis of the mortality rate may contribute to increased risk of death in Asians compared to the White population. ${ }^{[1]}$ Also, the African American population has a 2.8 fold higher death rate from COVID-19 compared to the White population. ${ }^{[2]}$ For instance, African Americans form 14 percent of the Illinois population, and African American COVID-19 deaths account for 36 percent of total

'ERBAS Institute of Experimental Medicine, Illinois, USA \& Gebze, Turkey 2Department of Physiology, Medical Faculty of Demiroğlu Bilim University, Istanbul, Turkey

Correspondence: İbrahim Gökçe. Deneysel Tıp Enstitüsü, 41470 GebzeKocaeli, Türkiye.

E-mail: ibrahimgokce84@gmail.com

Cite this article as: Gökçe I, Aydemir K, Ayan S, Altuntaş İ, Erbaş O. Effects of Human Genetic Factors (Ethnicity and Race) on Clinical Severity of SARS-CoV-2 (COVID-19). JEB Med Sci 2020;1(3):147-158.

doi: $10.5606 /$ jebms.2020.75631

Received : November 19, 2020

Accepted : November 27, 2020

Published online : December 29, 2020

(C2020 Journal of Experimental and Basic Medical Sciences. All rights reserved.

\begin{abstract}
Coronavirus Disease-19 (COVID-19) which is caused by SARS-CoV-2 (Severe acute respiratory syndrome coronavirus 2) has been declared as a pandemic by the World Health Organization (WHO) on 11 March 2020. After the declaration, SARS-CoV-2 took its place in the scientific area. It is a striking fact about COVID-19 pandemic that mortality, morbidity, and severity of the infection differ from region to region and even from country to country. The reason for this case is that interaction between COVID-19 and human cell receptors, inflammation, complement, and coagulation responses in humans differs as a consequence of ethnicity and genetic diversity. In this review, the effects of gene variations dependent on ethnicity on the clinical course of SARS-CoV-2 is aimed to analyze.
\end{abstract}

Keywords: African Americans, Arab, Armenian, Asian, Black, COVID-19, ethnicity, genetic, Hispanic, Jewish, Race, SARS-CoV-2, Turkish, white.

confirmed COVID-19 deaths. ${ }^{[3]}$ This data, also, demonstrates a high death rate from COVID-19 infection in African Americans. Moreover, death rate per a hundred thousand population and casefatality ratio of several countries is demonstrated (Table 1 and Figure 1).

\section{SARS-COV-2 (COVID-19)}

Coronaviruses are zoonotic viruses that can infect many creatures, including humans and animals. Coronaviruses are from the Orthocoronaviridae subfamily of the Coronaviridae family. The strain known as SARS-CoV-2 from the $B$ lineage of betacoronavirus, which is in four coronavirus genera, is called COVID-19. ${ }^{[4]}$ SARS-CoV-2, a-CoV group coronavirus, is a positive-oriented and single-stranded enveloped RNA virus. ${ }^{[5]}$ It contains a glycoprotein called spike. A viral RNA encodes 4 structural proteins, 3 of which are membrane proteins. Membrane proteins 
Table 1. SARS-COV-2 death rate per a hundred thousand population of some countries

\begin{tabular}{lc} 
Countries & Death rate per $100 \mathrm{~K}$ population \\
\hline Belgium & 172.48 \\
Spain & 108.80 \\
United Kingdom & 100.23 \\
Italy & 124.66 \\
France & 97.28 \\
Sweden & 85.70 \\
Netherlands & 67.94 \\
Ireland & 46.54 \\
USA & 107.46 \\
Switzerland & 90.96 \\
Turkey & 26.10 \\
Indonesia & 8.49 \\
Canada & 42.85 \\
Portugal & 69.23 \\
Peru & 118.08 \\
Brazil & 93.58 \\
Germany & 41.95 \\
Denmark & 23.70 \\
Iran & 67.90 \\
Nigeria & 0.67 \\
Israel & 38.45 \\
Uganda & 0.64 \\
Qatar & 8.81 \\
United Arab Emirates & 7.05 \\
Mexico & 100.81 \\
Bulgaria & 109.31 \\
Syria & 4.31 \\
\hline htps/cora & \\
\hline
\end{tabular}

https://coronavirus.jhu.edu/data/mortality accessed [19.12.2020].

are S-glycoprotein (S), matrix protein (M), and envelope protein $(E)$. The other structural protein is the nucleocapsid $(\mathrm{N})$ around the RNA, Figure $2 .{ }^{[4]}$
These structural proteins play an important role in the entry of the virus into host cells and subsequent virus replication. Spike protein is a transmembrane glycoprotein protruding from the viral surface that determines the diversity of coronaviruses and host tropism. These proteins play an important role in the entry of coronavirus into the cell. Spike glycoprotein is composed of two subunits which are S1 and S2. S1 protein subunits and angiotensin-converting enzyme-2 (ACE2) receptor interaction is required for coronavirus to invade human cells. Therefore, the S1 subunit binds to the human ACE2 receptor (hACE2) whereas the S2 subunit fuses with the cell membrane. ACE2 and TMPRSS2 enzymes are required for viral infectivity, since another host enzyme, the serine protease TMPRSS2 (transmembrane serine protease 2) enzyme, supports the cellular entry of SARS-CoV-2. Immune responses, including the production of antibodies against structural proteins, are required to limit the spread of infection. With the S-glycoprotein found in SARS-CoV-2, it gains a crown-like structure in electron microscopy. In addition, this glycoprotein structure enters the cell by binding to cellular receptors such as TMPRSS2 and furin, known as host cell proteases, especially ACE2, on the surface of human cells of the virus. TMPRSS2, which is expressed from the upper respiratory epithelium cells, activates the SARS-CoV-2 spike protein and enables SARS-CoV-2 to enter the body by breaking down the ACE2 receptor that allows the virus to bind to the epithelium.$^{[5]}$ The viral affinity of the host cells is directly related to the severity of the disease because the host cell activates the S1 and S2 subunits of the S protein with the protease of the

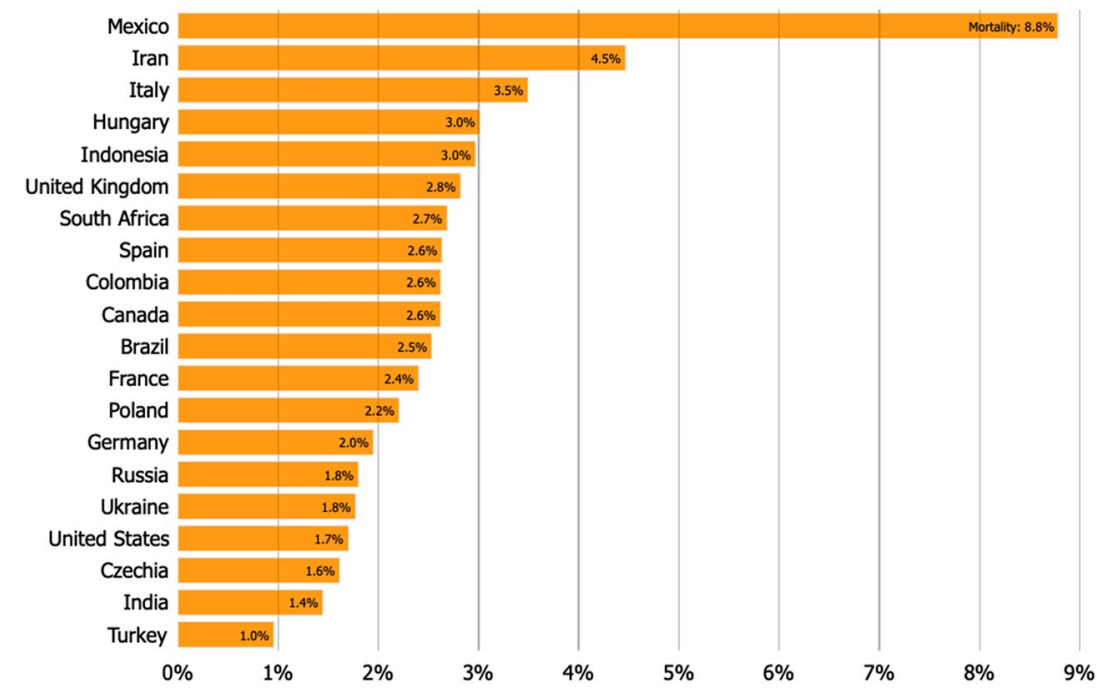

Figure 1. Mortality: Observed case-fatality ratio. 


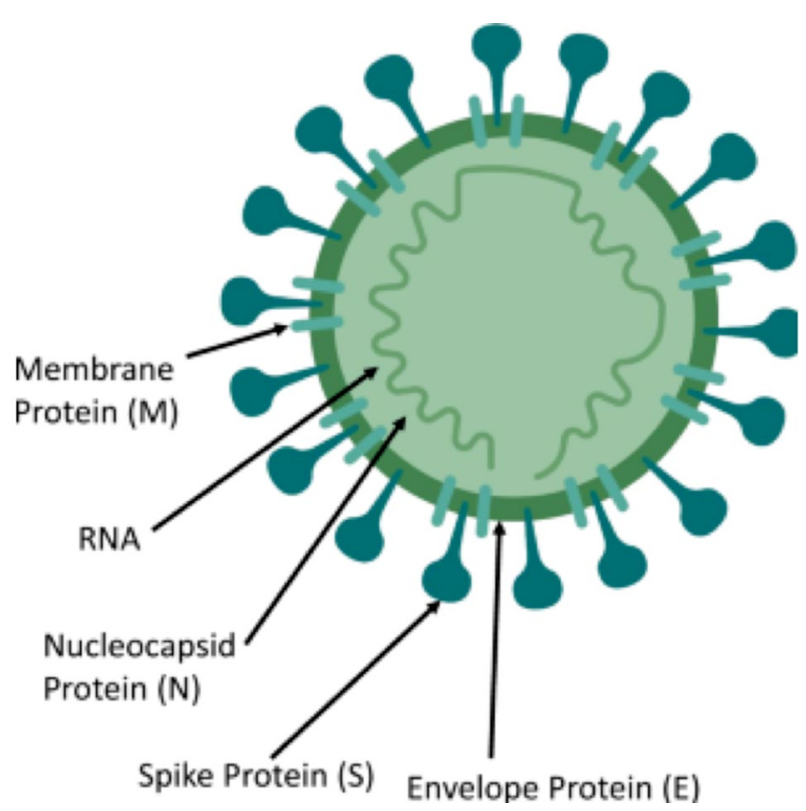

Figure 2. The structure of coronavirus.

virus. ACE2 is an enzyme found in the bronchi and bronchioles in the respiratory system. Therefore, SARS-CoV-2 replicates in the lower respiratory tract and is transmitted through droplets from symptomatic and asymptomatic infected people. ${ }^{[6]}$

Physiologically, ACE2 receptors are expressed in nasal, oral, nasopharynx, lung, gastric, duodenal, rectal, leydig, spermatogonial, sertoli epithelial cells. Active replication of infectious SARS-CoV-2 particles in human intestinal enterocytes due to the expression of the ACE2 receptor causes the virus to spread in the feces. Studies have reported that SARS-CoV-2 patients have gastrointestinal problems and symptoms such as diarrhea. ${ }^{[4,7]}$

SARS-CoV-2, defined as Severe Acute Respiratory Syndrome, was declared a pandemic by the World Health Organization (WHO) on March 11, 2020. SARS-CoV-2, a newly discovered coronavirus, is an infectious disease caused by the coronavirus. Some of the most common symptoms of SARSCoV-2 disease among humans are high fever, cough, as well as ground-glass in the lung in computed tomography (CT) images, Figure $3{ }^{[8]}$

Characteristic laboratory features include lymphopenia, high levels of C-reactive protein (CRP), lactate dehydrogenase (LDH), and aspartate aminotransferase (AST) ${ }^{[9]}$ SARS-CoV-2 risk groups include underlying factors such as advanced age, male gender, high body mass index (BMI),

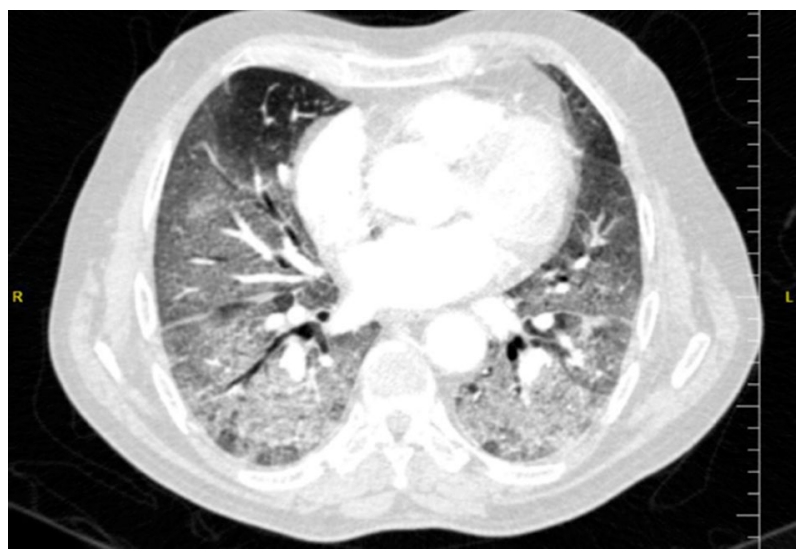

Figure 3. Ground-glass computed tomography image of patient with COVID-19. ${ }^{[8]}$

cardiovascular disease, chronic obstructive pulmonary disease (COPD), obesity, hypertension, diabetes, and chronic respiratory disease. ${ }^{[10]}$

\section{Biological Factors That May Cause Differences in Prevalence and Severity of SARS-CoV-2 (COVID-19) Infection}

\section{ANGIOTENSIN-CONVERTING ENZYME 2 (ACE2) GENE VARIANTS}

ACE2 expression has been associated with the clinical course of SARS-CoV-2 (COVID-19). The occurrence of ACE2 expression in various tissues such as the heart, lungs, kidneys, and intestines explains the situation of COVID-19 patients with multiple organ dysfunction. A number of studies have shown that Type II and Type I alveolar epithelial cells are the main source of ACE2 expression. In addition, endothelial cells were also determined to be ACE2 positive. Ethnogenetic factors, air pollution, smoking, age, and gender alter ACE2 expression. ACE2 expression increases in the respiratory tract with aging. Since the gene encoding the ACE2 enzyme is inherited from the $X$ chromosome, an increase in expression is observed in the respiratory tract since there is no other response of the $X$ chromosome in males. For this reason, infection and mortality rates are higher in the elderly and men. ${ }^{[11]}$

In the studies, it was determined that six genes (SLC6A20, LZTFL1, CCR9, FYCO1, CXCR6, and XCR1) located in the locus of the 3rd chromosome (3p21.31) are associated with the clinical severity of SARSCoV-2. Among these genes, SLC6A20 and LZTFL1 have been shown to be intensely expressed in the human lung and are closely related to the course of the 
disease. The SLC6A20 gene is known to encode the sodium-iminoacid (proline) transporter-1 (SIT-1) gene. In conjunction with ACE2, this gene plays a role in the adhesion of the virus to the cell surface and its entry into the cell. In addition, this gene locus encodes proteins such as the chemokine receptor-9 (CCR9) and the C-X-C motif chemokine receptor- 6 (CXCR6) region. These chemokine receptors are known to be associated with the intensity of the lung infectioninduced immune response and the $T$ lymphocyte response. ${ }^{[12]}$

In another study conducted by Stawiski et al., it is examined whether natural variations of ACE2 in the population affect human ACE2's affinity to novel coronavirus (2019-nCoV/SARS-CoV-2). ${ }^{[13]}$ Findings stated that S19P, I21V, E23K, K26R, K26E, T27A, N64K, T92I, Q102P, M383T, and H378R are the polymorphic variants of ACE2 which increase interaction between human ACE2 and SARS-CoV-2 S-protein; any other way, K31R, N33I, H34R, E35K, E37K, D38V, Y50F, N51S, K68E, F72V, Y83H, G326E, G352V, D355N, and Q388L variants are estimated to have detractive effects on SARS-CoV-2 S-protein and human ACE2 interaction. ${ }^{[13]}$ Even though K26R variant is stated as an enhancer of S-protein and ACE2 receptor binding, recent articles indicate it in the reverse way. For another perspective, there are some determined variants that may affect SARS-CoV-2 S-protein and human ACE2 binding affinity in both negative and positive ways, these are; S19P, T27A, K31R, N33I, H34R, E35K, E37K, D38V, N51S, N64K, K68E, F72V, T921, Q102P, G326E, G352V, D355N, H378R, Q388L, and D509Y. ${ }^{[13]}$

\section{EFFECT OF K341R AND G211R VARIANTS}

K341R variant has been proven as an enhancer of SARS-CoV-2 - ACE2 binding and this variant is most frequently found in African Americans. ${ }^{[14]}$ One of the reasons for the high death rate of African Americans could be associated with this situation. Moreover, G211R variant of ACE2 which is abundant in the Asia population (about $0.2 \%$ ) has demonstrated as having the most interaction with SARS-CoV-2 S-protein. ${ }^{[14]}$ Therefore, it could also be thought as the reason for the high death rate seen in Asian population.

\section{EFFECT OF K26R VARIANT}

The K26R (rs4646116) variant is an ACE2 variant that facilitates the binding of the S-glycoprotein effective in binding the coronavirus to the host cell and increases the susceptibility to the virus. The K26R variant, which reduces virus binding to the ACE2 receptor, has been found to be more concentrated in the Jewish population (1.2\%) and, in contrast, the Asian population has the lowest allele frequency for the single nucleotide variant encoding K26R. ${ }^{[15]}$

\section{EFFECT OF N720D VARIANT}

Transmembrane serine protease (TMPRSS), trypsin, FURIN, and plasminogen, referred to as host cell proteases play a very important role in viral entry by triggering the binding of the virus to the receptor. Transmembrane serine proteases 2 (TMPRSS2), which plays a substantial role in breakdown and activation of viral glycoprotein, is an important factor in determining severity of viral infection. Camostat methylate, an inhibitor of TMPRSS2 protease activity has been shown that it is able to reduce the entry of SARS-CoV-2 into human lung epithelial cells and a promising candidate for potential intervention against SARS-CoV-2. N720D is a kind of ACE2 variant that increases TMPRSS 2 activation and facilitates entry of the virus into host cells. This variant is rare in the Middle East and Africa, but not in the East Asian population. ACE2 modification is performed by TMPRSS2, and this modification leads to increased S-protein viral entry. Since N720D is associated with the TMPRSS2 modification site, the N720D mutation affects TMPRSS2 and may increase the effect of SARS-CoV-2 as a result of the increased binding of the virus. ${ }^{[16]}$ The N720D (rs41303171) variant is $2.5 \%$ in Europeans, $0.6 \%$ in Iranians, $0.3 \%$ in Kuwait and $0.2 \%$ in Qatar populations. These rates have been evaluated in relation to low mortality in Kuwait and Qatar. ${ }^{[15]}$

Another protein closely related to ACE2 is FURIN. FURIN variation in the Middle East region (Qatar and Kuwait) is thought to be protective against SARS-CoV-2. ${ }^{[15]}$

\section{ACE1 I/D (INSERTION/DELETION) ALLELE FREQUENCY}

ACE I/D polymorphism has been found associated with the clinical course of SARSCoV-2. ${ }^{[17]}$ In a study, the association between ACE1 I/D genotype and mortality and morbidity of SARS-CoV-2 infection is examined in Asia and Europe. ${ }^{[17]}$ ACE1 II polymorphism has been found to be associated with lower death and infection rate of SARS-CoV-2 infection. ${ }^{[17]}$ It is determined the European population has a lower frequency of ACE1 Il genotype, and this situation might explain higher mortality and prevalence rates of COVID-19 infection in the European region. In another study, ACE I/D polymorphism data collected from the population 


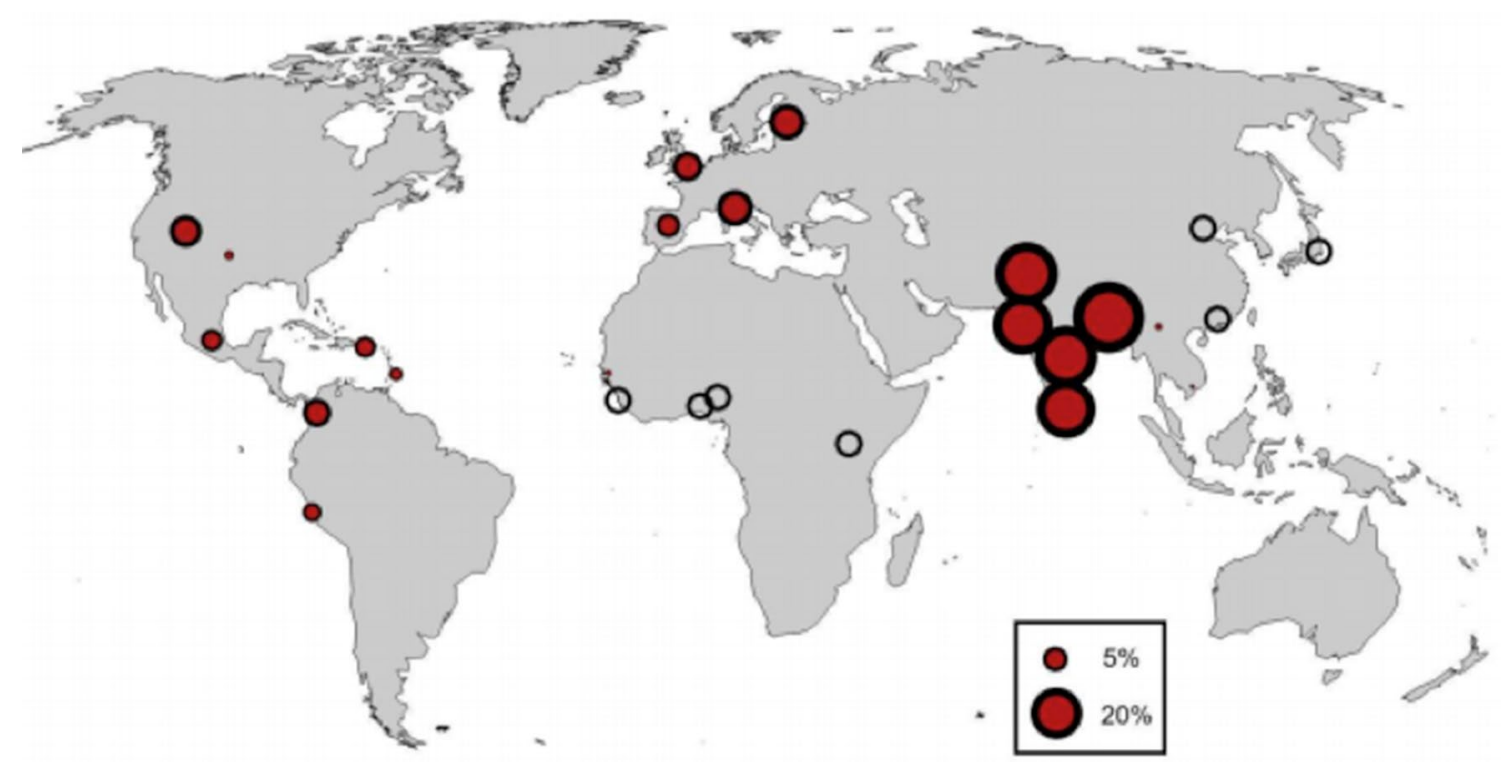

Figure 4 Neanderthal Core Haplotype Distribution. (Hollow circles indicate the populations which are lacking Neanderthal haplotype). ${ }^{[21]}$

of 38 Asian countries, and results are analyzed. Linear correlation between ACE D allele frequency and number of cases infected with SARS-CoV-2 has been found in the Asian population. ${ }^{[18]}$ Since ACE I/D polymorphism has been associated with high levels of $A C E$, it can be interpreted that high levels of ACE expression may have negative outcomes on SARSCoV-2 infection. ${ }^{[18]}$

\section{ADAM 17 ACTIVITY}

ADAM 17 (a disintegrin and metalloproteinases) is a protein involved in the removal of some membrane proteins important for immunity and inflammation, such as tumor necrosis factor-alpha, intercellular adhesion molecule-1, and ACE-2. It was observed that ADAM17 expression and activity increased in patients with sepsis. ${ }^{[19]}$ ADAM 17 polymorphisms in South Asians are thought to affect ACE-2 levels and the inflammatory response to viral infection.

\section{NEANDERTHAL HERITAGE GENES}

Neanderthal is mostly of European and Asian origin and this gene is not found in the African region. The susceptibility of people from different parts of the world to infection varies and this is due to our genes inherited from ancestors. ${ }^{[20]}$ In the epizootiology of COVID-19, the genetic basis is the presence of six genes with neandertal inheritance on chromosome 3 at locus 3p21.31. The Neanderthal haplotype is $30 \%$ in South Asia, $8 \%$ in Europe, 4\% among mixed Americans, and lower in East Asia. Therefore, Neanderthal variants may contribute crucially to COVID-19 infection risk in certain populations, Figure $4 .{ }^{[21]}$

\section{microRNA (miRNA)}

MiRNAs are small non-coding RNAs (about 22 nucleotides) known to regulate post-transcriptional expression that affect different cellular stages such as cell differentiation, apoptosis, and proliferation. Some researchers have identified miRNAs that affect ACE2-related nephropathy. A unique miRNA called hsa-miR-27b has been found in the SARS-CoV-2 genome isolated from India. It is known that this micro RNA inhibits ACE expression and will increase ACE2 expression due to the inverse relationship between them. ${ }^{[22]}$

\section{OCCURRENCE OF VIRAL MUTATIONS}

While SARS-CoV-2 genome obtained from India has demonstrated a unique mutation in viral Spike surface glycoprotein, this mutation is not encountered in other strains in the USA, Wuhan, Italy, and Nepal. ${ }^{[2]}$

\section{ANDROGENS}

As a result of orchiectomy performed in rats (surgical removal of one or both testicles), a decrease 
in ACE2 expression was found. ${ }^{[23]}$ The low androgen levels in South Asian men compared to Caucasian men in the United States can be associated with SARS-CoV-2.

\section{HUMAN LEUKOCYTE ANTIGEN (HLA) POLYMORPHISM}

Human leukocyte antigen (HLA) alleles play an active role in viral susceptibility and in changing the severity of the disease. Nguyen et al. ${ }^{[2]}$ claim that genetic variability among HLA-A, B, C may have a crucial role in susceptibility to and severity of the novel coronavirus (2019-nCoV/SARS-CoV-2). In the disease caused by the SARS-CoV virus, which is similar to SARS-CoV-2, an increase is observed in individuals carrying the HLA-B*46: 1 genotype. ${ }^{[25]}$

Figure 5 demonstrates global distribution maps of HLA allele frequency for the six HLA $-A,-B$, - $C$ alleles. HLA-A*25:01, HLA-B*46:01, HLA-C*01:02 alleles are identified to have the fewest estimated binding peptides for 2019-nCoV peptides that means people with these three genotypes may have more susceptibility to and severe infection of SARS-CoV-2. ${ }^{[24]}$ On the other hand, HLA-A*02:02, HLA-B*15:03, HLA-C*12:03 alleles are identified to have the most estimated bindings peptides for SARS-CoV-2 peptides and this situation points out that individuals with these three genotypes may have less susceptibility and severe infection of SARS-CoV-2. ${ }^{[24]}$

\section{X-CHROMOSOMAL TOLL-LIKE RECEPTORS (TLRS) AND IFN ANTIBODY RESPONSE}

Toll-like receptors (TLRs) belong to a group of pattern recognition receptors (PRCs) which are a crucial part of innate immunity. Currently, 12 different TLRs have been defined in mammals. ${ }^{[26]}$ It has been shown that TLR7 gene is responsible for expression of TRL7 receptor. TLRs are associated
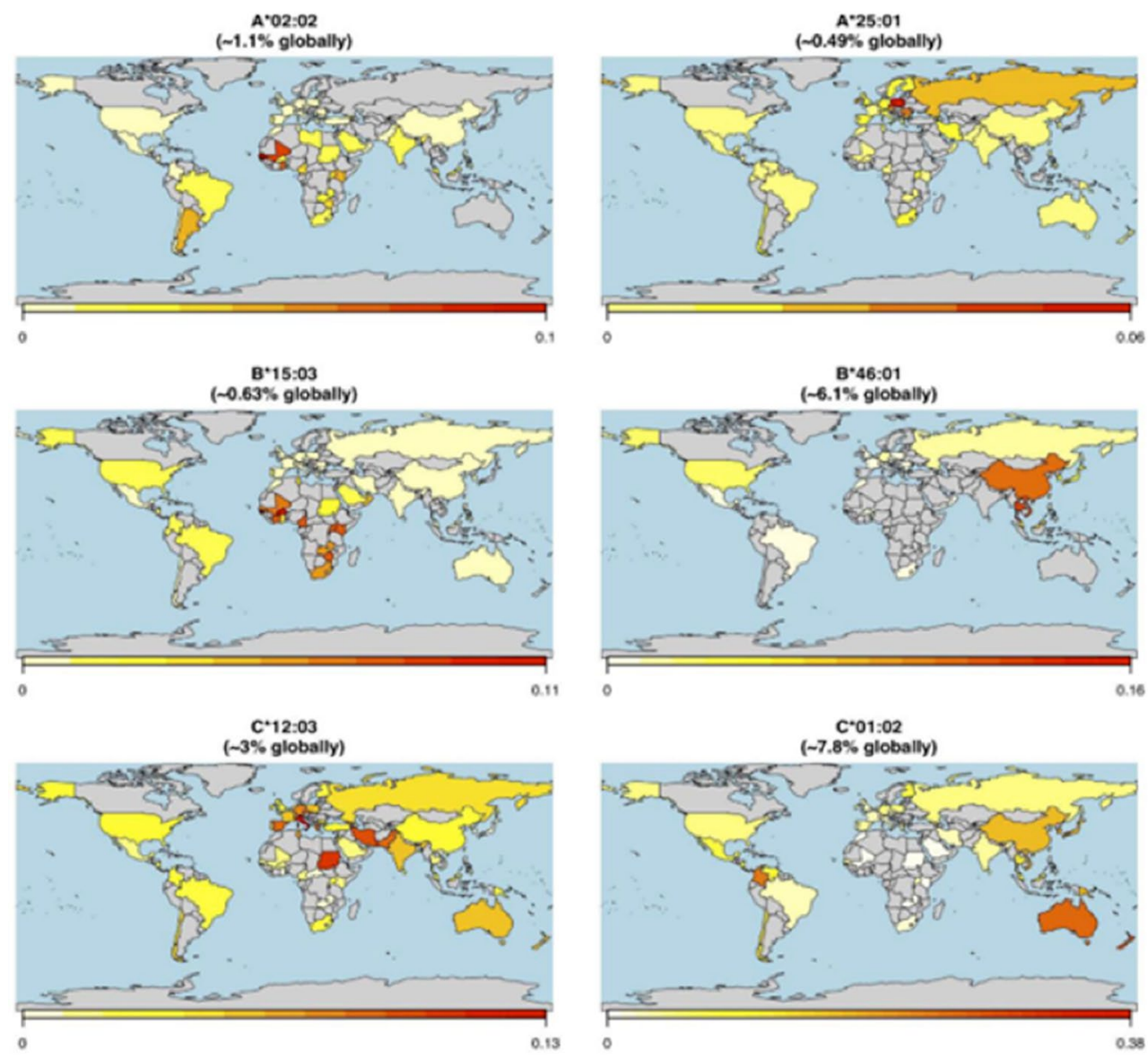

Figure 5. Global distribution map of HLA allele frequency. 
with interferon I and II (IFN I and II) response that play essential roles in fighting against the infections and modulating immune response. ${ }^{[27,28]}$ Since TLR polymorphism varies according to ethnicity, it is thought that this situation may cause susceptibility to SARS-CoV-2. ${ }^{[29]}$ In a study conducted in the Netherlands, under 35 years old two brothers infected severely with SARS-CoV-2 from unrelated two families have been chosen and their IFN I and II response related TLR7 gene analysis is achieved by whole-exome sequencing (WES) technique. As a consequence of the analysis, it is determined that all of the four individuals have the variants of X-chromosomal TLR7 gene which causes loss of function. ${ }^{[28,30]}$ Once the situation of the cases is analyzed, it is found that all patients needed mechanical ventilation in intensive care service (ICU) and additionally, one case resulted in death. ${ }^{[30]}$ While four nucleotide deletions in TLR7 gene (c.2129_2132del; p.[GIn710Argfs*18]) are determined in the family of two brothers having one death, a missense mutation in TLR7 gene (c.2383G >T; p.[Val795Phe]) causing amino acid sequence change is determined in the other family. ${ }^{[30]}$ It is obviously observed also from other studies that TLR7 is a crucial part of innate immunity against coronavirus infections including SARSCoV-2 infection. ${ }^{[30]}$ In the study of under 35 years old four people having X-chromosomal TLR7 variants that cause loss of function exhibit TLR7 variants can be used as a biomarker to determine susceptibility to COVID-19 infection and more comprehensive studies are required to reach precise consequences.

\section{PREVIOUS EXPOSURE TO MALARIA}

Malaria is a serious contagious disease result in severe health conditions and even deaths. It is spread by a certain type of mosquito feeding on humans. Constant exposure to malaria forms tolerance to proinflammatory response against SARS-CoV-2 infection, and as a result, the occurrence of blunted cytokine response is possible. ${ }^{[31]}$ It has been shown that there is a negative correlation between the number of SARS-CoV-2 cases and malaria per million population. ${ }^{[32]}$

\section{BCG VACCINATION}

While the investigation on vaccines and specific therapies for COVID-19 has been continuing, the protective effects of the Bacille Calmette-Guérin (BCG) vaccine on infection have recently been discussed in preprints. ${ }^{[33]}$ Normally, BCG vaccine composed of inactivated Mycobacterium bovis is used to provide protection against Mycobacterium tuberculosis infection. ${ }^{[34]}$ Also, it is well known that BCG vaccination policy is applied by countries that have high Tuberculosis (TB) incidence. However, recently, protective effects of BCG vaccination on SARS-CoV-2 infection have been discussed in the scientific area. Madan et al. demonstrate in their retrospective cross-sectional study that lower incidence of COVID-19 infection is determined in the countries which have a high-TB burden. ${ }^{[35]}$ Moreover, in the same study, they discuss countries having a low-TB burden due to BCG vaccination policy may be seen as a protective effect of BCG vaccination on COVID-19 infection. ${ }^{[35]}$ The reason for this situation may be clarified by cross-immunity among mycobacterium species and COVID-19. ${ }^{[35]}$ Also, epidemiological explorations suggest that BCG vaccination may take place in reducing the severity of COVID-19 infection. ${ }^{[34]}$ Therefore, the possibility of $B C G$ vaccine providing resistance against the harmful effects of SARS-CoV-2 is emphasized. But there are also countries like Brazil that are exceptions to this.

\section{INTERLEUKIN-6 (IL-6) POLYMORPHISM}

An important member of the cytokine network is IL- 6 and plays a central role in acute inflammation. The biological activity of IL- 6 is determined by its potential to activate target genes that regulate cell differentiation, survival, proliferation, and apoptosis. ${ }^{[36]}$ Increased levels of Interleukin-6 (IL-6) in SARS-CoV-2 patients have been associated with disease severity. It has been stated that the $C$ allele, which is a polymorphism of the IL- 6 gene, is more intense in Caucasians than Indians, therefore IL- 6 production is higher in Caucasians. ${ }^{[36]}$

\section{LACK OF VITAMIN-D}

A study conducted by Nurshad Ali emphasizes the association between vitamin-D level and COVID-19 cases and deaths in 20 European countries. ${ }^{[37]}$ Although there is an inverse relationship between the average vitamin-D level per million population and COVID-19 cases in European countries, no significant relationship was found between Vitamin-D level and COVID-19 death rates. ${ }^{[37]}$ Furthermore, the role of vitamin-D in reducing the threat of pneumonia and acute respiratory tract infections has been shown in several types of research. ${ }^{[37]}$ The reducing effect of vitamin-D could be explained by several mechanisms. One of the mechanisms is triggering cathelicidins and defensins which take play in lowering the viral 
replication rates and decreasing pro-inflammatory cytokines. ${ }^{[38]}$ Furthermore, it may be considered as an important factor that COVID-19 pandemic started in winter in the North Hemisphere and in summer in the South Hemisphere. The angle of incidence of sunshine is dependent on seasons. Since the activation of vitamin-D in the human body is activated by sunshine, the vitamin-D levels of populations can change according to the season they are in. It is determined that 25 -hydroxyvitamin $\mathrm{D}(25(\mathrm{OH}) \mathrm{D})$ concentrations of the population are in the lowest degree in winter. ${ }^{[38]}$ Lower numbers of COVID-19 cases in the South Hemisphere until the end of the summer may be explained by the effect of $25(\mathrm{OH})$ D concentration. ${ }^{[38]}$ However, there is no sufficient evidence to mention the association between vitamin-D level and the clinical course and mortality of COVID-19 cases, further investigation should be emphasized to reach precise consequences.

\section{ABO BLOOD TYPE}

Previously published reports have suggested an association between $A B O$ blood groups, cardiovascular disease, cancer, and some viral infections. ${ }^{[39]}$ It is clear that blood type causes sensitization in viral infections caused by Norwalk and Hepatitis B viruses. ${ }^{[40]}$ In addition, it has been reported that people with the 0 blood group are less susceptible to being infected in the infection caused by the SARS coronavirus, which emerged in 2003. ${ }^{[40]}$ Observing relationships between $A B O$ blood groups and genetic predisposition in previous viral infections made us think that the same relationship could also be in COVID19 and paved the way for studies on this issue. Blood samples were collected and analyzed from 2,173 patients diagnosed with COVID-19 by realtime polymerase chain reaction (PCR) test from three different hospitals in Wuhan and Shenzhen, China. ${ }^{[40]}$ In the study in which 1,775 patients (206 of whom were dead) from Wuhan Jinyintan Hospital were analyzed, it was observed that the rate of blood group $A$ in patients diagnosed with COVID-19 was higher than the distribution in the normal population, and on the contrary, the rate of $\mathrm{O}$ blood group in patients diagnosed with COVID-19 was lower than the distribution in the normal population. Similarly, this distribution was found in dead cases. ${ }^{[40]}$

As a result of these studies, it can be stated that individuals with blood type $A$ are in the highrisk group against SARS-CoV-2 infection, on the contrary, individuals with blood group $\mathrm{O}$ have a lower risk of the same infection. Based on this study, it can be deduced that ABO blood groups can be a biomarker to determine susceptibility to COVID-19. In addition, the effects of Rh factor in addition to $A B O$ blood groups were examined and when the data of a multi-institutional study and a study conducted with cases from the New YorkPresbyterian (NYP) hospital system were examined, it was found that the prevalence of infection was higher in Rh-positive individuals. ${ }^{[39,41]}$

There are also studies in the literature that examine the relationship between $A B O$ blood group and COVID-19 from a different perspective. Unlike other studies in the literature, in a study by Gerard et al., an approach to analyzing the effects of anti-A and anti-B antibodies (found in blood groups 0 and $B$ ) was adopted instead of focusing on the antigens of $A B O$ blood groups. ${ }^{[42]}$

In the study, it was thought that anti-A antibody circulating in the blood could interfere with viruscell binding and inhibit virus-cell binding, and it was found that individuals with anti-A antibodies in blood serum were significantly less represented in the COVID-19 group. ${ }^{[42]}$

\section{APOE GENE}

The apolipoprotein E (APOE) gene is a gene responsible for the synthesis of a protein called apolipoprotein $E$, and in extensive studies (metaanalyses), the APOE gene has been found to be associated with different neurological pathologies, especially dementia. ${ }^{[43]}$ The APOE gene has various alleles such as $\varepsilon 2, \varepsilon 3, \varepsilon 4$. With the worldwide impact of the COVID-19 pandemic, studies conducted in the last year have also found that carriers of the Apoc4 allele, especially in the older age group, have increased sensitivity to SARS-CoV-2 infection. ${ }^{[4]]}$ The fact that morbidities such as diabetes and dementia, which increase the risk of COVID-19 infection, are also associated with variants in the APOE gene cluster may be important in determining susceptibility to COVID-19 infection. ${ }^{[4]}$ Apos4 homozygous individuals $(\varepsilon 4 / \varepsilon 4)$ were found to have a 2.2 -fold higher risk of being infected with COVID-19 than Apos3 homozygous $(\varepsilon 3 / \varepsilon 3)$ individuals and also had a 3.4-fold higher case/death rate. ${ }^{[4]}$ When heterozygous individuals $(\varepsilon 3 / \varepsilon 4)$ are examined, the lower level of association with COVID-19 suggests that the effect of the Apos4 allele on COVID-19 outcomes is recessive. ${ }^{[44]}$ 


\section{CROSS IMMUNITY}

Cross immunity can be defined as protection against pathogens due to adaptive immunity from previous exposure to similar pathogens. When this situation is adapted to SARS-CoV-2 infection, it can be said that the adaptive immune response resulting from previous exposure to other coronavirus infections such as SARS-CoV and MERS-CoV infections may protect against SARS-CoV-2 infection. ${ }^{[45]}$ The reason for the low mortality rate in Asia and the Middle East can be explained by the adaptive immune response because Asian and Middle Eastern populations have been repeatedly exposed to coronavirus infections. ${ }^{[45]}$ In addition, $T$ cell reactivity to SARSCoV-2 coronavirus has been shown to be present in $20 \%$ to $50 \%$ of individuals known to be not exposed to SARS-CoV-2 in various types of research. ${ }^{[46]}$ This result also contributes to the concept of crossprotective immunity. Therefore, more research should be studied to comprehensively understand the cross-protective immune effect on SARS-CoV-2 infection.

\section{CD209L/L-SIGN AND CD209/DC-SIGN}

CD209 receptors are located in the innate immune system and can recognize a variety of pathogens. ${ }^{[47]}$ In recent studies, it is claimed that CD209 is associated with the SARS-CoV-2 S-protein and mediates the entry of SARS-CoV-2 into human cells. ${ }^{[48]}$ CD-209 and CD209L receptors mediate this process by binding the receptor binding domain (RBD) of the SARS-CoV-2 virus. ${ }^{[48]}$ CD209L expression is prominently detected in lung and kidney epithelium and endothelial cells of vessels, while CD209 expression is observed in limited cell types. ${ }^{[48]}$ In the light of the information given, it can be suggested that CD209 and CD209L receptors can be considered as alternative receptors for the SARS-CoV-2 coronavirus. Therefore, it may be promising to examine these mechanisms in detail for the antiviral treatment of SARS-CoV-2 infection.

\section{THE MEDITERRANEAN FEVER (MEFV) GENE}

The Mediterranean Fever (MEFV) gene, located on the $16^{\text {th }}$ chromosome, is mainly expressed by monocytes and granulocytes. ${ }^{[49]}$ These cells are involved in inflammatory pathophysiology and cytokine storm. ${ }^{[49]}$ MEFV gene, which has more than 300 sequence variants, was previously thought to be associated with protective roles in some diseases such as tuberculosis, smallpox, cholera, plague, typhus, and malaria. ${ }^{[50]}$ It can be stated that mutation carriers of the MEFV gene are more protected in some diseases than those that do not carry the mutation. ${ }^{[50]}$ Kavukçu and Soylu ${ }^{[50]}$ published in it is emphasized that the lower mortality rate from COVID-19 in some populations may be due to the increased prevalence of MEFV mutation carriers seen in these populations. ${ }^{[50]}$ In the COVID-19 weekly surveillance report, these populations with lower COVID-19 incidence and mortality are noted as Jewish, Arab, Turkish and Armenian populations. ${ }^{[51]}$ In addition, the role of MEFV mutations in COVID-19 disease was investigated in 50 COVID-19 patients diagnosed with PCR test. ${ }^{[49]}$ As a result of the analysis of MEFV gene mutations using Sanger sequencing in these patients, it was found that 6 patients (12\%) had heterozygous MEFV mutations. ${ }^{[49]}$ According to previous studies, the prevalence of MEFV mutations in this region was 25 percent. ${ }^{[49]}$ In the light of the information given, it can be observed that MEFV mutation carriers are at a significantly lower incidence in COVID-19 patients compared to the normal population distribution in the region. ${ }^{[49]}$ In addition, populations of the eastern Mediterranean regions with a high carrier rate of MEFV mutations have a slightly lower prevalence of COVID-19. ${ }^{[49]}$ Considering the data in the study, it can be claimed that MEFV mutations may have protective effects on the mortality and morbidity of SARS-CoV-2 infection.

\section{DISCUSSION AND CONCLUSION}

There are a variety of ethnicities all around the world and these ethnicities lead to genetic diversity which affects the clinical course of diseases and response to treatments. In light of this information, it can be interpreted that which ethnicities will have the diseases at which rate. By using the same logic, it could be extracted that ethnicity and genetic diversity can be helpful in recognizing the disease and treatment processes of SARS-CoV-2 infection. Based on the comprehensive studies on clinical course of SARS-CoV-2 and ethnicity association, it can be suggested that Asian, African Americans and Hispanic populations are at high death risk of SARS-CoV-2 infection. Furthermore, the same interpretation can be done for the risk of becoming infected with SARS-CoV-2 in these populations.

On the other hand, the spread rate of COVID-19 infection in Africa and South 
America is demonstrated to be associated with socioeconomic and social living standards as a result of research. Citizens of nations that have low per capita income can not take balanced and healthy diets. Depending on the malnourishment in these people, physiologic and hormonal metabolisms of the human body can not fulfill their functions properly. Additionally, unplanned pregnancy leads to plenty of population and this situation causes a competitive environment. Moreover, limited job opportunities cause future anxiety and psychological problems in individuals. Malnourishment and psychological problems collapse the immune system of individuals and as a result of it, disease rates in the community whose members have problematic immune systems increase. Furthermore, it is an undeniable reality that Africa and South America regions deal with crucial hygiene problems.

Southeastern Asia and Africa have been known as the starting point of major pandemics throughout history. Consumption of raw living organisms has caused a variety of diseases and the Asian population has been living with these diseases for long years. Lack of clean water and hygienic conditions in Africa also contributes to the spread of various diseases. Therefore, it appears in some research that people having this ethnic origin can become immune genetically to these pandemics.

As mentioned in these studies, any disease can not be assessed independently from individuals genetic backgrounds, lifestyles, and environmental conditions. Individuals are not able to arrange their genetic background but they are able to decide their environment and lifestyles. Therefore, an appropriate environment and healthy lifestyles could be a prescription for a healthy life.

\section{Declaration of conflicting interests}

The authors declared no conflicts of interest with respect to the authorship and/or publication of this article.

\section{Funding}

The authors received no financial support for the research and/or authorship of this article.

\section{REFERENCES}

1. Sze S, Pan D, Nevill CR, Gray LJ, Martin CA, Nazareth $J$, et al. Ethnicity and clinical outcomes in COVID-19: A systematic review and meta-analysis. EClinicalMedicine 2020;29:100630.

2. Deaths involving coronavirus disease 2019 (COVID19) by race and Hispanic origin group and age, by state. 2020. Available at: https://data.cdc.gov/NCHS/ Deaths-involving-coronavirus-disease-2019-COVID-19/ ks3gspdg

3. Doumas M, Patoulias D, Katsimardou A, Stavropoulos K, Imprialos K, Karagiannis A. COVID19 and increased mortality in African Americans: socioeconomic differences or does the renin angiotensin system also contribute? J Hum Hypertens 2020;34:764-7.

4. Ludwig S, Zarbock A. Coronaviruses and SARS-CoV-2: A Brief Overview. Anesth Analg 2020;131:93-6.

5. Astuti I, Ysrafil. Severe Acute Respiratory Syndrome Coronavirus 2 (SARS-CoV-2): An overview of viral structure and host response. Diabetes Metab Syndr 2020;14:407-12.

6. Salzberger B, Buder F, Lampl B, Ehrenstein B, Hitzenbichler F, Hanses F. Epidemiologie von SARS-CoV-2-Infektion und COVID-19 [Epidemiology of SARS-CoV-2 infection and COVID-19]. Internist (Berl) 2020;61:782-8.

7. Noorimotlagh Z, Karami C, Mirzaee SA, Kaffashian M, Mami S, Azizi M. Immune and bioinformatics identification of $T$ cell and $B$ cell epitopes in the protein structure of SARS-CoV-2: A systematic review. Int Immunopharmacol 2020;86:106738.

8. Mohamed YG, Mohamud MFY, Medişoğlu MS, Atamaca IY, Ali IH. Clinical and chest CT presentations from 27 patients with COVID-19 pneumonia in Mogadishu, Somalia: a descriptive study. Egypt J Radiol Nucl Med 2020;51:184.

9. Lai CC, Shih TP, Ko WC, Tang HJ, Hsueh PR. Severe acute respiratory syndrome coronavirus 2 (SARSCoV-2) and coronavirus disease-2019 (COVID-19): The epidemic and the challenges. Int J Antimicrob Agents 2020;55:105924.

10. Ziegler CGK, Allon SJ, Nyquist SK, Mbano IM, Miao VN, Tzouanas CN, et al. SARS-CoV-2 Receptor ACE2 Is an Interferon-Stimulated Gene in Human Airway Epithelial Cells and Is Detected in Specific Cell Subsets across Tissues. Cell 2020;181:1016-35.e19.

11. Williamson EJ, Walker AJ, Bhaskaran K, Bacon S, Bates C, Morton CE, et al. Factors associated with COVID-19-related death using OpenSAFELY. Nature 2020;584:430-6.

12. Severe Covid-19 GWAS Group, Ellinghaus D, Degenhardt F, Bujanda L, Buti M, Albillos A, et al. Genomewide Association Study of Severe Covid-19 with Respiratory Failure. N Engl J Med 2020;383:1522-34.

13. Stawiski EW, Diwanji D, Suryamohan K, Gupta R, Fellouse FA, Sathirapongsasuti JF, et al. Human ACE2 receptor polymorphisms predict SARS-CoV-2 susceptibility. bioRxiv 2020.

14. Ali F, Elserafy $M$, Alkordi MH, Amin M. ACE2 coding variants in different populations and their potential impact on SARS-CoV-2 binding affinity. Biochem Biophys Rep 2020;24:100798.

15. Al-Mulla F, Mohammad A, Madhoun AA, Haddad D, Ali H, Eaaswarkhanth $\mathrm{M}$, et al. A comprehensive germline variant and expression analyses of ACE2, TMPRSS2 and SARS-CoV-2 activator FURIN genes from the Middle East: Combating SARS-CoV-2 with precision medicine. bioRxiv 2020. 
16. Klaassen K, Stankovic B, Zukic B, Kotur N, Gasic V, Pavlovic $S$, et al. Functional prediction and comparative population analysis of variants in genes for proteases and innate immunity related to SARS-CoV-2 infection. Infect Genet Evol 2020;84:104498.

17. Yamamoto N, Ariumi Y, Nishida N, Yamamoto R, Bauer G, Gojobori T, et al. SARS-CoV-2 infections and COVID-19 mortalities strongly correlate with ACE1 I/D genotype. Gene 2020;758:144944.

18. Pati A, Mahto H, Padhi S, Panda AK. ACE deletion allele is associated with susceptibility to SARS-CoV-2 infection and mortality rate: An epidemiological study in the Asian population. Clin Chim Acta 2020;510:455-8.

19. Shao Y, He J, Chen F, Cai Y, Zhao J, Lin Y, et al. Association Study Between Promoter Polymorphisms of ADAM17 and Progression of Sepsis. Cell Physiol Biochem 2016;39:1247-61.

20. Gill RS, Dogra M. COVID-19: A Geo-Anthropogenic Analysis. International Journal of Science and Research 2020;9:1555-66.

21. Zeberg $H$, Pääbo $S$. The major genetic risk factor for severe COVID-19 is inherited from Neanderthals. Nature 2020;587:610-2.

22. SardarR, Satish D, Birla S, Gupta D.Comparative analyses of SAR-CoV2 genomes from different geographical locations and other coronavirus family genomes reveals unique features potentially consequential to host-virus interaction and pathogenesis. bioRxiv 2020.

23. Dalpiaz PL, Lamas AZ, Caliman IF, Ribeiro RF Jr, Abreu GR, Moyses MR, et al. Sex Hormones Promote Opposite Effects on ACE and ACE2 Activity, Hypertrophy and Cardiac Contractility in Spontaneously Hypertensive Rats. PLoS One 2015;10:e0127515.

24. Nguyen A, David JK, Maden SK, Wood MA, Weeder BR, Nellore A, et al. Human Leukocyte Antigen Susceptibility Map for Severe Acute Respiratory Syndrome Coronavirus 2. J Virol 2020;94:e00510-20.

25. Ceraolo C, Giorgi FM. Genomic variance of the 2019nCoV coronavirus. J Med Virol 2020;92:522-8.

26. Kumar H, Kawai T, Akira S. Toll-like receptors and innate immunity. Biochem Biophys Res Commun 2009;388:621-5.

27. Lee AJ, Ashkar AA. The Dual Nature of Type I and Type II Interferons. Front Immunol 2018;9:2061.

28. van der Made $\mathrm{Cl}$, Simons A, Schuurs-Hoeijmakers J, van den Heuvel G, Mantere T, Kersten S, et al. Presence of Genetic Variants Among Young Men With Severe COVID19. JAMA 2020;324:663-673.

29. Gupta R, Misra A. COVID19 in South Asians/Asian Indians: Heterogeneity of data and implications for pathophysiology and research. Diabetes Res Clin Pract 2020;165:108267.

30. Anastassopoulou C, Gkizarioti Z, Patrinos GP, Tsakris A. Human genetic factors associated with susceptibility to SARS-CoV-2 infection and COVID-19 disease severity. Hum Genomics 2020;14:40.

31. Tian X, Li C, Huang A, Xia S, Lu S, Shi Z, et al. Potent binding of 2019 novel coronavirus spike protein by a
SARS coronavirus-specific human monoclonal antibody. Emerg Microbes Infect 2020;9:382-5.

32. Sala G, Miyakawa T. Association of BCG vaccination policy with prevalence and mortality of COVID-19. medRxiv 2020.

33. Kumar J, Meena J. Demystifying BCG Vaccine and COVID19 Relationship. Indian Pediatr 2020;57:588-9.

34. Escobar LE, Molina-Cruz A, Barillas-Mury C. BCG vaccine protection from severe coronavirus disease 2019 (COVID19). Proc Natl Acad Sci U S A 2020;117:17720-6.

35. Madan M, Pahuja S, Mohan A, Pandey RM, Madan K, Hadda $V$, et al. TB infection and BCG vaccination: are we protected from COVID-19? Public Health 2020;185:91-2.

36. Kesarwani $P$, Ahirwar D, Singh R, Manchanda PK, Mittal RD. Do IL-4 intron 3 VNTR and IL-6 (-174) G/C variants reflect ethnic variation? A comparative study between the global and North Indian populations. Asian Pac J Cancer Prev 2008;9:76-80.

37. Ali N. Role of vitamin D in preventing of COVID-19 infection, progression and severity. J Infect Public Health 2020;13:1373-80.

38. Grant $W B$, Lahore $H$, McDonnell SL, Baggerly CA, French $C B$, Aliano $\mathrm{J}$, et al. Evidence that Vitamin $D$ Supplementation Could Reduce Risk of Influenza and COVID-19 Infections and Deaths. Nutrients 2020;12:988.

39. Latz CA, DeCarlo C, Boitano L, Png CYM, Patell R, Conrad MF, et al. Blood type and outcomes in patients with COVID-19. Ann Hematol 2020;99:2113-8.

40. Zhao J, Yang Y, Huang H, Li D, Gu D, Lu X, et al. Relationship between the ABO Blood Group and the COVID-19 Susceptibility. Clin Infect Dis 2020:ciaa1150.

41. Zietz M, Zucker J, Tatonetti NP. Testing the association between blood type and COVID-19 infection, intubation, and death. medRxiv [Preprint] 2020:2020.04.08.20058073.

42. Gérard C, Maggipinto G, Minon JM. COVID-19 and $\mathrm{ABO}$ blood group: another viewpoint. $\mathrm{Br} J$ Haematol 2020;190:e93-e94.

43. Forero DA, López-León S, González-Giraldo $Y$, Dries $D R$, Pereira-Morales AJ, Jiménez KM, et al. APOE gene and neuropsychiatric disorders and endophenotypes: $A$ comprehensive review. Am J Med Genet B Neuropsychiatr Genet 2018;177:126-42.

44. Finch CE, Kulminski AM. The ApoE locus and COVID-19: are we going where we have been? J Gerontol A Biol Sci Med Sci 2020:glaa200.

45. Yaqinuddin A. Cross-immunity between respiratory coronaviruses may limit COVID-19 fatalities. Med Hypotheses 2020;144:110049.

46. Doshi P. Covid-19: Do many people have pre-existing immunity? BMJ 2020;370:m3563.

47. Chen L, Zheng S. Understand variability of COVID-19 through population and tissue variations in expression of SARS-CoV-2 host genes. Inform Med Unlocked 2020;21:100443.

48. Amraie R, Napoleon MA, Yin W, Berrigan J, Suder E, Zhao G, et al. CD209L/L-SIGN and CD209/DC-SIGN act as receptors for SARS-CoV-2 and are differentially 
expressed in lung and kidney epithelial and endothelial cells. bioRxiv 2020.

49. Salehzadeh F, Shahbazifar E, Pourfarzi F, Molatefi R, Davarnia B, Ahmadabadi, F. The role of MEFV gene in COVID 19 disease, as a protective factor. 2020. Available at: https://www.researchsquare.com/article/rs-69373/v1.

50. Kavukçu S, Soylu A. Could MEFV mutation carriage status have a protective role for COVID-19 pandemic? Med Hypotheses 2020;144:109889.

51. WHO Weekly surveillance report-COVID-19. 2020. Available at: https://www.euro.who.int/en/healthtopics/health-emergencies/coronavirus-covid-19/ weekly-surveillance-report/weekly-surveillancereport-alt. 